\title{
Recruitment Search Engines for Screening Resumes through AI by Using Boolean Search Functions
}

\author{
Sunaina Arora \\ Research scholar, Faculty of Management Studies, \\ Manav Rachna International Institute of Research and Studies \\ Haryana, India \\ Prof. Dr. Neeraj Kumari (Corresponding Author) \\ Department of Applied Sciences, Faculty of Engineering \& Technology, \\ Manav Rachna International Institute of Research and Studies \\ Haryana, India \\ E-mail: neerajnarwat@gmail.com
}

Received: August 5, 2021 Accepted: October 12, $2021 \quad$ Published: October 29, 2021

doi: 10.52941/jad.v7i2.23 URL: https://doi.org/10.52941/jad.v7i2.23 


\section{Abstract}

The days are gone when hard copies of resumes were sent to recruiters and they used to screen and set aside non relevant resumes. Artificial Intelligence has taken up mundane tasks of recruiters by simplifying search algorithms and human computer interaction. Job Boards provide recruiters with a database of candidates powered by a search tool with lot of filters. The demonstration paper actually carries out searches on recruitment database access tools to filter the relevant applicants from a wide pool of data with the help of search engine tools in resume databases of various jobboards. It shows how Boolean operators get through better search results in few minutes. Recruiter job to get to the right candidate is sorted out with Artificial Intelligence so that they can focus more on strategic tasks. Future of AI in recruitment is immense. Already companies are empowering recruiters with 360 degree tools which help with all Human Resource Aspects.

Keywords: Recruitment Database, Boolean Search, Job Boards, Artificial Intelligence

\section{Introduction}

The days of the employing of random job seekers after scrutinizing through classifieds and other registered job boards have gone by. Identify the best talent in this age of a dynamic environment, the norm is a suitable requisite. Finding the best talent is the challenge. Several workers are terribly unable to find the best talent available in the market for a job requisition. There is no regular expertise in talent mapping. It takes a lot of patience and stamina because recruiting specialists take lot of pieces of knowledge into account before delivering the entire picture. Recruitment practitioners have a comprehensive eye and are capable of mapping right talent if provided with the right recruitment tools.

Artificial Intelligence (AI) has most fundamental advantage thatit replicates the choices and actions of people without human innate vulnerabilities, such as energy lack, emotion, partiality and short time. It is also simpler for multiple AI computers to attain consistent efficiency than for multiple human employees to do the same. The evidence says that AI has the ability to speed up recruiting function to speed up the recruitment process, remove repetitive, manual tasks from the profile of the workers, and thereby make them more efficient.The opportunity to spot the information needed in a relatively small span of time from a broad databaseis where AI is needed for the recruiting process. As far as the recruiting process is concerned, AI saves hiring managers time as well as expenses. It eliminates the need to source talent from third-party service providers. Thus, it is possible to do away from job offices and conventional recruiting firms and the expenses involved. AI is designed to automate some portion of the recruiting workflow in the recruiting sector, especially routine, high-volume activities, namely the recruiting workflow. Sourcing, scanning, resume reviews, and interview preparation. It is the recruitment departments that fail to attract applicants inside the talent management teams; procurement continues to be the most difficult aspect of the operation. It is also the most time-consuming part, because of the procedure, about $80 \%$ of the resumes received for a category are declined at various different 
stages of the hiring process.AI can also be used to scan and retrieve information from resumes that are publicly accessible on the internet, to interpret the details and decide which applicants are ideally fit for each job profile.

Recruitment Databases are the main source of readily available pool of talent. These database apply numerous filters to get to the exact match of candidate which the employer is specifically looking at.

In India major job boards like Naukri.com, Monster.com, Timesjobs.com and Shine.com widely collect data of employees and market them to employers in a subscription based model. All can be done when there is a pool of data available with AI and ML, from matching candidates to work, user experience, providing them advice / suggestions, finding hot skills, providing recruiter with the right match.

Whereas Linkedin focusses on providing social engagement platform to jobseekers and employers, build their own professional network and approach candidates available in their network. There is a free search feature which is available to all Linkedin users. There is also a paid subscription to search relevant candidates through various filters using Boolean operators.

Effortless automatic shortlisting,displaying relevant applicants, reaching candidates through emails, scheduling interviews and background verification are the jobs performed by currently AI powered recruitment tools.

\subsection{Literature Review}

To determine the values, interests and behaviours of the candidate, AI will search social media information of a candidate. AI recruiters not only get resumes but get clues about personality characteristics and suitability. AI is impartial, and the screens restart equally by giving both contestants equal weight. AI is transforming the recruiting business and is eliminating routine activities that human recruiters have historically done. As, recruiters are happy to assign the tedious, repeated roles to AI-powered programs, AI is making certain processes redundant. AI is changing the positions played by recruiters. They now have the time to be imaginative and will work on strategic problems, with AI taking care of tedious and routine activities. (Upadhyay \& Khandelwal, 2018)

In the recruiting process, the novelty aspect of using AI mediates and further positively affects career applicant probability. There are some major functional effects of these favourable associations between attitudes towards the use of AI in the recruiting process and the risk of applying for a position. First, it suggests that while anxiety is naturally present while AI is part of the recruiting process, the anxiety does not actually impact the fulfilment of work applicants, so companies do not need to expend resources on either shielding their use of AI or reducing the rate of anxiety of prospective employees. On the opposite, the study indicates that in fear of alienating potential applicants, organisations may not need to conceal their use of AI. But in the hiring process, companies tend to promote their use of AI and focus on prospective recruits who 
already have positive views of both the enterprise and Artificial intelligence. (Van Esch, Black, \& Ferolie, 2019)

The quest task also involves formulating complicated queries that are represented as Boolean strings. Creating powerful Boolean queries, remains a critical task, frequently hampered by mistakes and shortfalls. (Russell-Rose, Chamberlain, \& Kruschwitz, 2019)

Automating the recruiting processes through AI will actually free recruiters from mundane tasks so as to have the time to become more human. They can utilize this free time to more deeply explore the needs and wants of potential candidates and determine whether they fit into the organization.

In order to have the time to be a little more human, streamlining the recruitment processes through AI would eventually free hiring managers from basic tasks. They will use this extra time to investigate the interests and preferences of potential participants more thoroughly to decide whether they are right fit for the organisation.

The potential to spot the relevant information in a relatively short timespan from a broad repository is where the procurement process requires the deployment of AI. Tasks such as submitting follow-up messages, calling applicants when accurate job profiles are open, notifying personnel when an applicant is actively searching for work, can be easily done by AI.This allows companies without large teams to ramp up their recruiting processes. In general, AI provides enterprises with tools that helps them to establish a bond rather than only gathering resumes from their prospective future hires. (Wilfred, 2018)

While Artificial Intelligence helps to make recruiting tasks more effective and apparently less discriminatory by integration, it relies entirely on human-generated data, and may therefore have subconscious human bias translated to model which is embedded in AI through humans. (Mujtaba \& Mahapatra, 2019)

There are many successful initiatives that work to improve chances of getting a job on a worldwide scale by creating a suitable career for ideal job seekers. (Keršič, Štukelj, Kamišalić, Karakatić, \& Turkanović, 2019)

AI saves time, which results in not doing the repetitive tasks. It saves time as resume scanning is a tedious process. Technology works out to attain the correct aspirant to the enterprise and the outsourcing work to recruiting firms is minimized. (Geetha \& Bhanu, 2018)

Recent trends show that technology or AI will replace routine human tasks and Managers will try to focus energy on strategic functions. Recruitment processes will be streamlined through AI and technology shift will be used to save time, cost and energy by shifting to AI. (Hmoud \& Laszl, 2019)

Recruitment and Selection practitioners appear to focus mainly on three: chatbots, Resume Screening softwares and methods for task automation. Tech-focused businesses tend to be the 
majority of companies implementing these AI tools. Considering the exponential pace of AI adoption, enterprises also have to hit an inflexion point because they are actually hesitant to invest in Recruitment \&Selection in technology. (Albert, 2019)

(Krishnakumar, 2019) Sometimes AI options could be discriminatory, and there is little empirical evidence on the fairness of AI recruiting schemes as the human element is absent.

\subsection{Objectives of the Study}

- To demonstrate how artificial intelligence is used in recruitment databases to screen relevant candidates

- To highlight use of Boolean operators in Resume Database search engines

- To understand the importance of AI in Recruitment

\section{Methodology of the Study}

The study was done usingIndia's Leading Naukri.com resume databasefree search, Timesjobsfree resume search tooland Linkedin free search. Some searches were performed in the search engine of the Database to see how the actual query generates right Resumes according to the keywords, experience, Employer Name, etc. All recruitment database over the world use the similar search features to search the right resumes.

\subsection{Analysis}

Recruitment Database Search Engines of Job Boards provide search tools to scrutinize resume pool of candidates. The Resumes can be shortlisted on the basis of keywords, experience range, Annual Salary, location, employer, functional area, Industry, Designation and many more appropriate filters.

In order to use the search function in resume database tools, the recruiter must know how and when to use Boolean operators to get to the best matching resumes in the shortest possible time. Employers need to know the job requirements specifically and also should know similar skills with different headers which jobseekers generally write in their resumes to perform a search query. For example sales is also written as business development in some resumes.

Boolean Operators are basic terms (AND, OR, NOT or AND NOT) used in a search with different combinations and permutations of keywords, the results are clustered in different ways and often give efficient outcomes or the best pool of candidates available in the database. By using not operator employers can filter non relevant resumes and save time. Proper usage comes with practice and knowledge about the alternate skills or keywords which are generally found in resumes of a particular set of candidates performing a certain role in organization. 


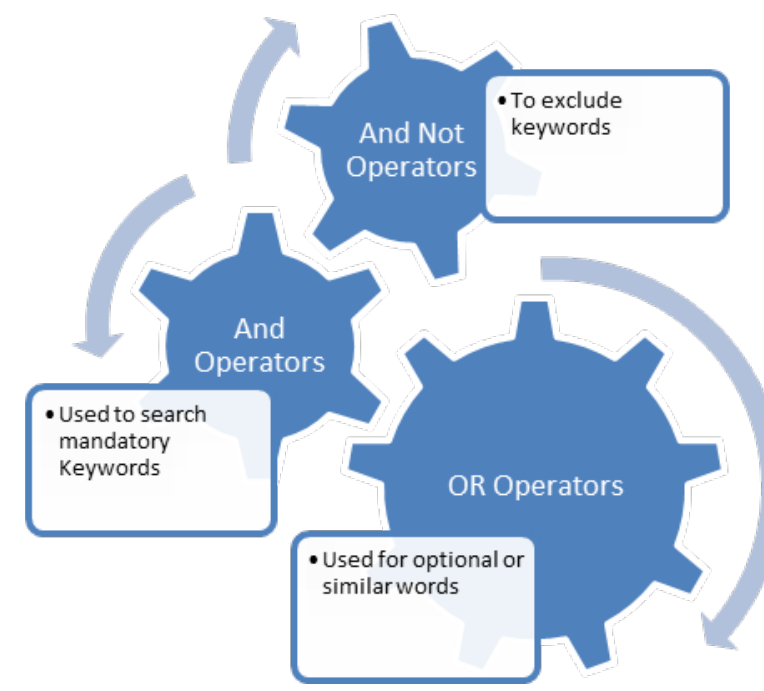

Figure 1. and, or, and not Operators (Source Self)

Interpretation: Figure 1 depicts the Use of Boolean strings in advance search features of recruitment databases;

AND Operator - Is used when all the keywords in the resume are mandatory i.e. the recruiter requires these skills or keywords to be present in the resume. Example: ('Data Scientist' and Python)

OR Operator - While searching resumes where optional or similar words are present OR operator is used. When recruiter thinks that ideal resume may have one or more such keywords, he mentions synonyms so that he does not miss on resumes which have mentioned synonyms are keywords. Example:('Data Scientist' or 'Data Science') and (Azure or Cloud)

AND NOT Operator- To exclude keywords which are not required in the resume. Example: ('Data Scientist' or 'Data Science') and (Azure or Cloud) and not (Java)

Use of Parenthesis- You should enclose search terms and their operators in parentheses to define the order in which they are read, to allow even greater use of Boolean operators. First, information is read within parentheses, and then information beyond parentheses is read next.

Use of Inverted Commas- To identify Keywords as a phrase we have to use inverted commas in the search box. Using Inverted commas is important because, if we are searching for 'Project Manager' the search engine would otherwise take Project and Manager as separate keywords and would not highlight right results.

(Russell-Rose \& Chamberlain, 2016) Recruiters have continued desire to structure searches as Boolean expressions, and recruitment search engines should manage, organise, and re-use search techniques and results. The ranking of the profiles is with respect to the search parameters which 
were set by the recruiter.

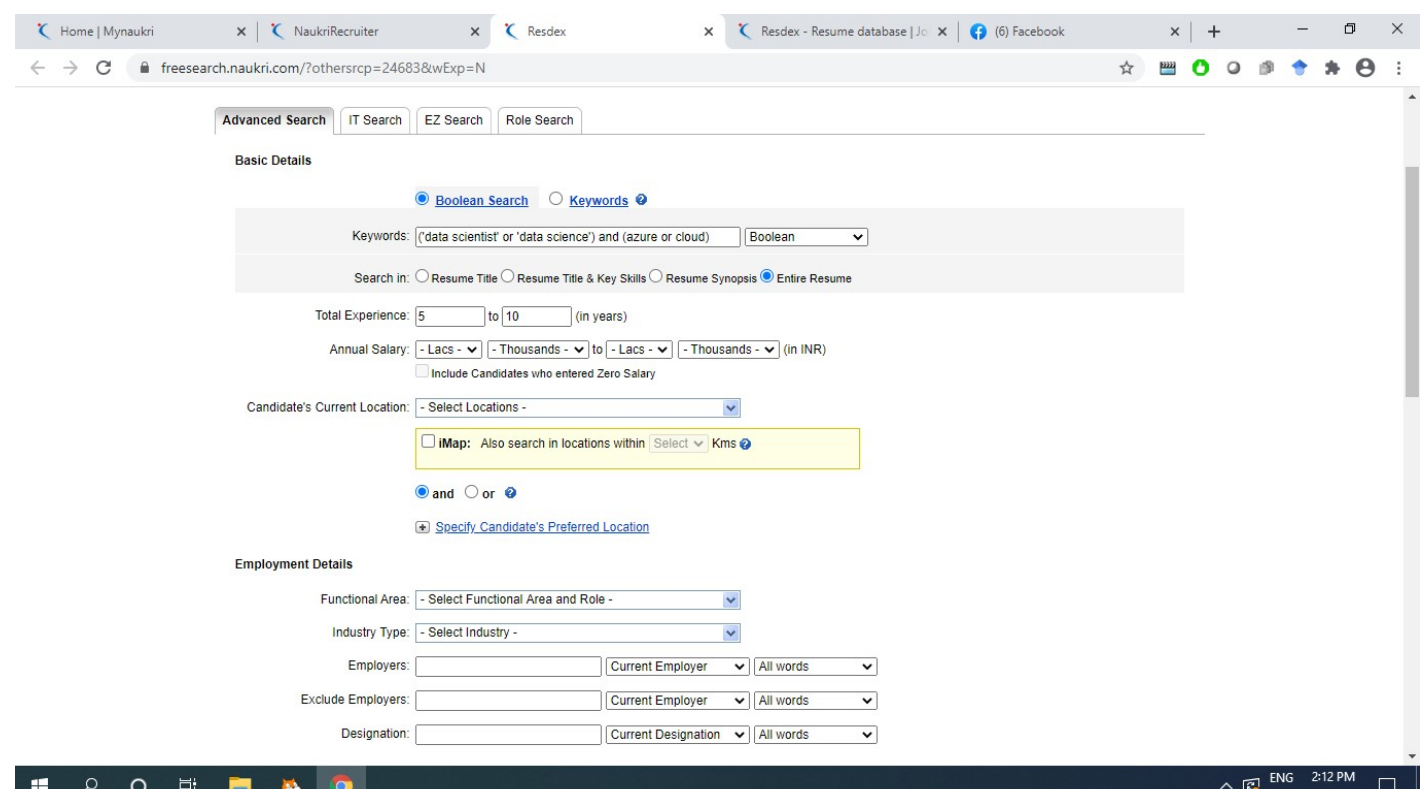

Figure 2. Resume Database Screening Tool, Source Naukri.com Free Search

Interpretation: The Figure 2; depicts the actual image taken from the Software application which screens a large pool of data of resumes. There are a lot of filters which can be used to narrow down the search. It eases out the work of recruiters to get to contact only the relevant candidates for the job position.

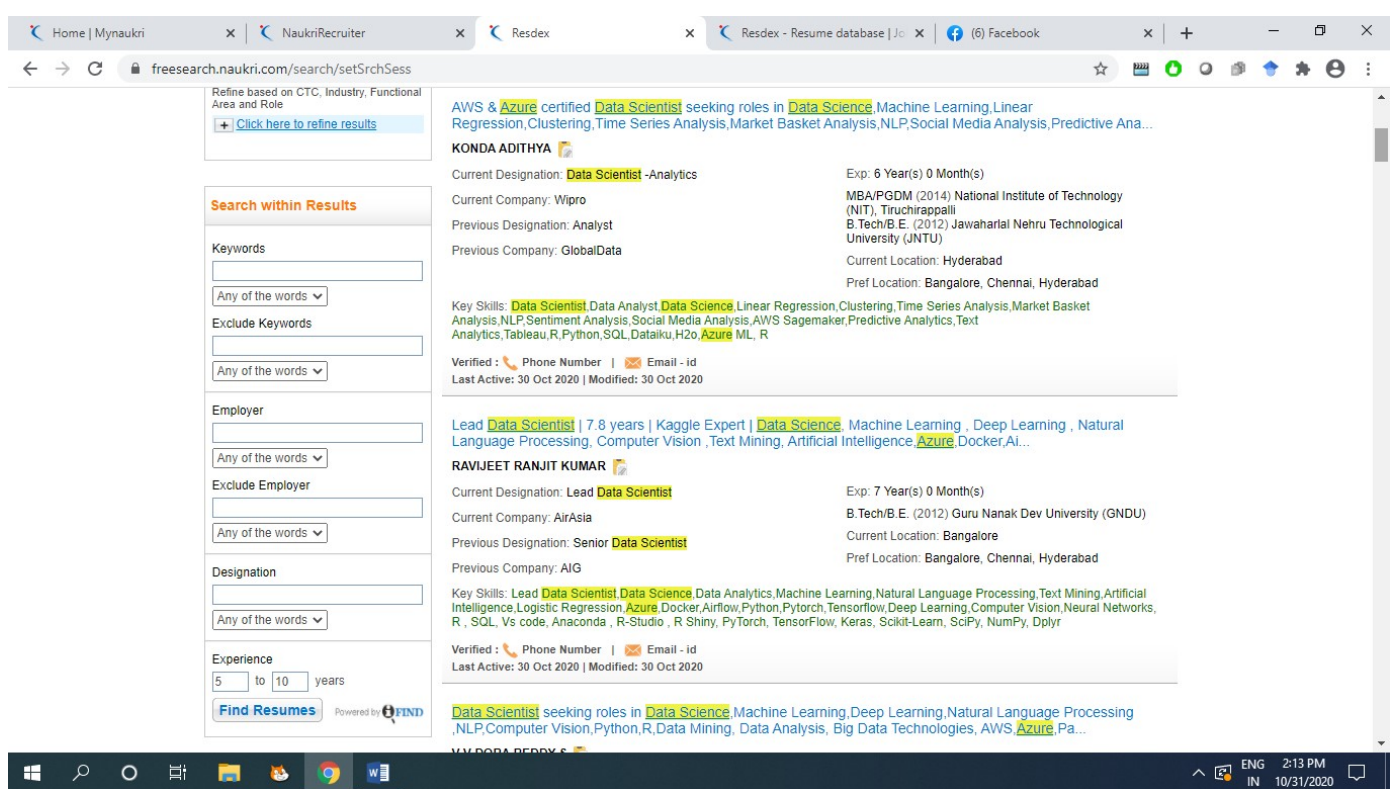

Figure 3. Actual Search Results after applying the filters; Source Naukri.com Free Search 
Interpretation: Figure 3; shows the actual image from the resume database access software on how it matches the candidates and shows up the relevant candidates to the recruiters. Saving a lot of time of recruiters. There are functions to contact the candidate through messages, phone or email.Keywords are highlighted.

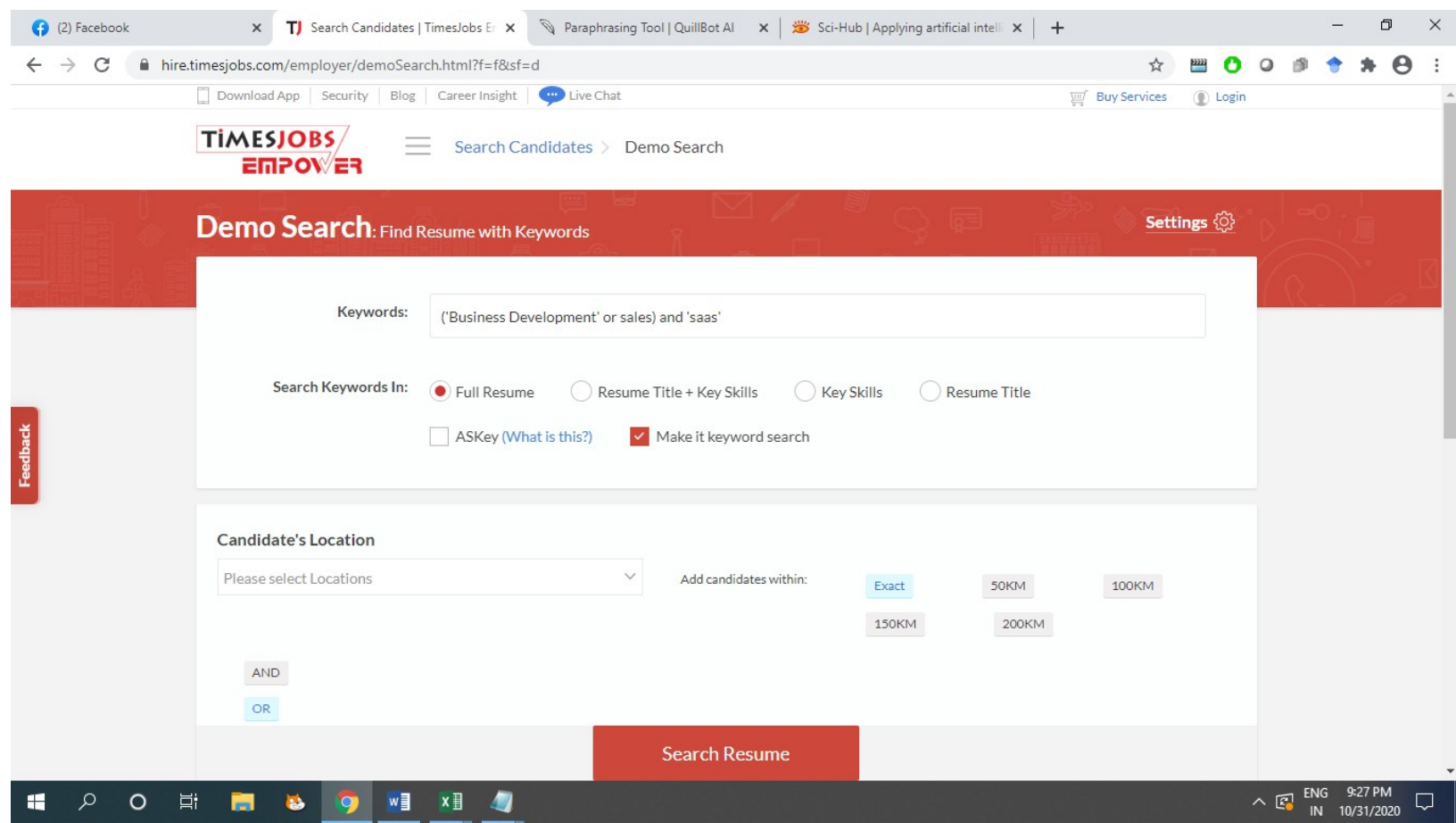

Figure 4. Actual Image from Timesjobs, database search engine tool, free search

Interpretation: Figure 4, Depicts actual image from the recruitment database software of timesjobs.com; the Tool shows various filters which help to filter resumes from the pool of candidates available on the job boards. 


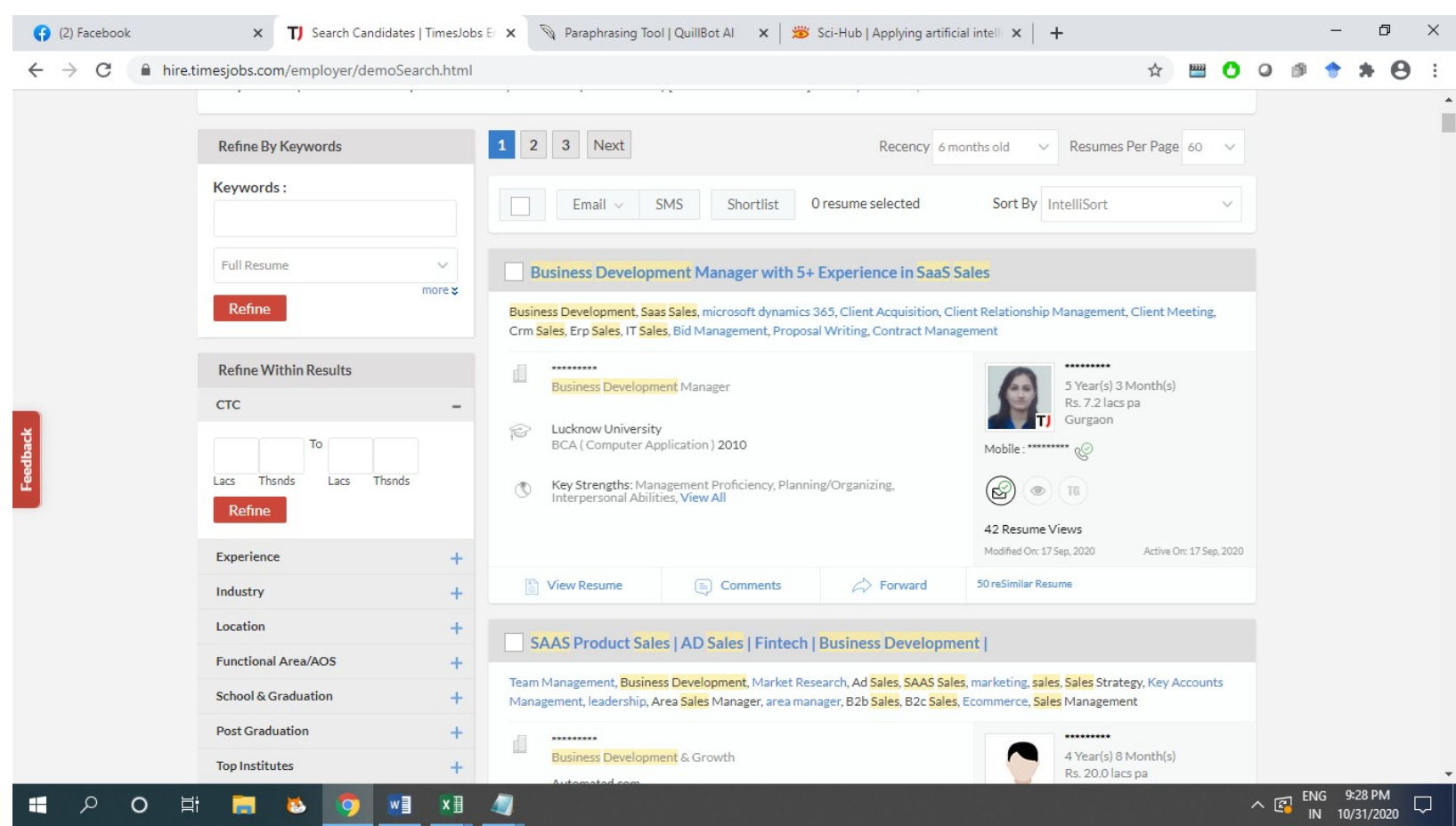

Figure 5. Actual Image from search results from timesjobs.com recruitment database search tool

Interpretation: Figure 5 shows how the software screens the resumes exactly according to the keywords mentioned in the search tool. It throws up relevant resumes according to the search action performed. Thus, easing the job of a recruiter.

But, the free search tool on Linkedin does not allow Boolean operators. Whereas, the paid version allows Boolean search and has a very useful project-based recruitment tracking AI.

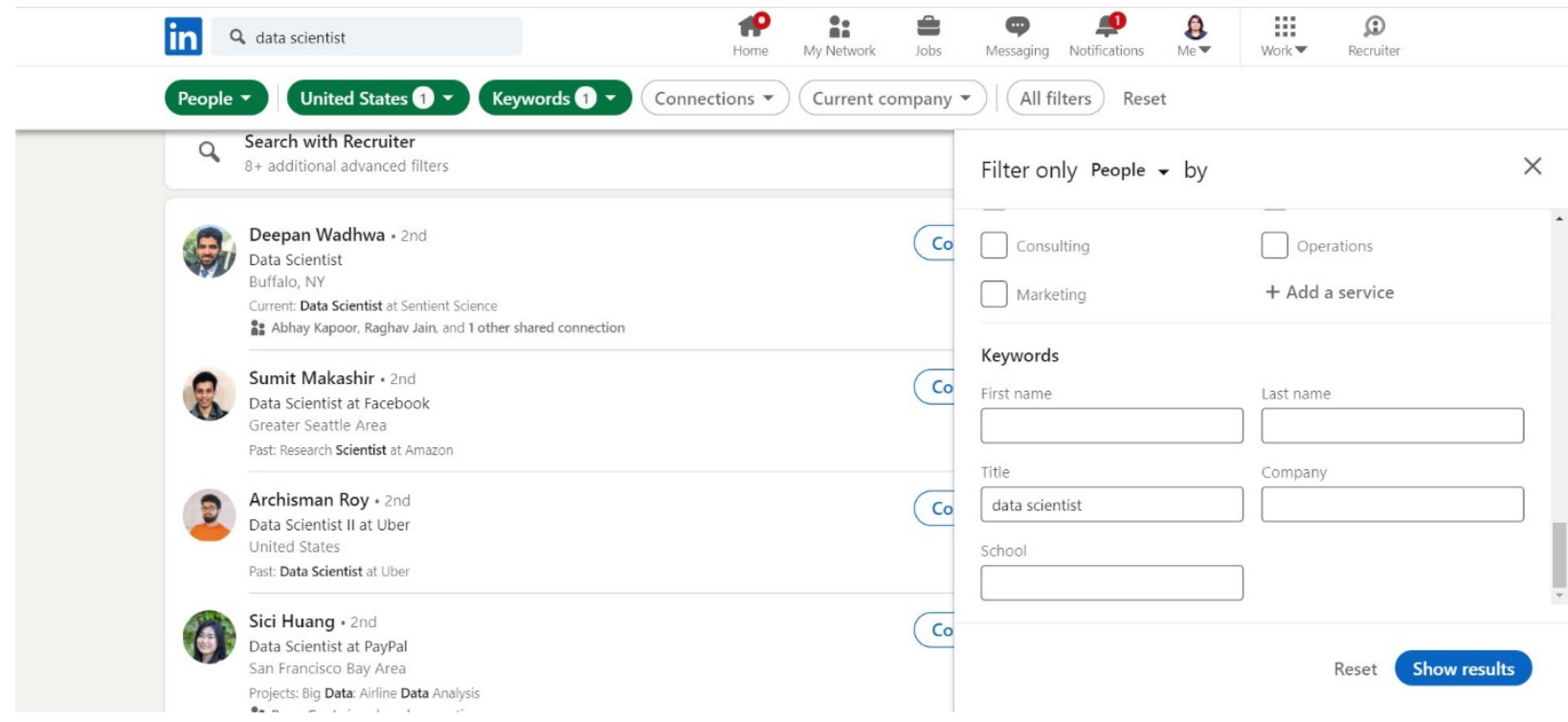

Figure 6. Actual image of the Linkedin free search tool 
Interpretation: Figure 6 depicts that boolean operator cannot be used in the free search but AI automatically shows the top companies to find data scientists in United States of America. The left-hand column automatically shows the profiles according to the keywords search in the title or the search bar.

\section{Conclusion}

The Recruitment Database software and their screening algorithms helps to save recruiters time and effort in mundane daily tasks of opening each and every resume to search for a right candidate. Rather it matches the Key Skills, Keywords, experience, location, Cost to company, employers, etc. and gets the best candidate out of the pool of resumes available in the database.

While using the recruitment database tools several Boolean searches were conducted which depicts the usage of Boolean operators in the search tools to find out the exact match resumes of candidates.

Artificial Intelligence and Machine Learning are essential tools required for mundane recruitment functions. The repetitive tasks of searching right candidates for a particular job requisition is administered within minutes with help of software and thus, recruiters can spend time on strategic activities which require more human interactions.

\subsection{Innovative Inputs to Improve Recruitment Search Engines Aiinthe Future}

Companies have taken recruitment search engines to a next level by recommending relevant candidate matches according to the history of searches created on their platform. Recruitment search engines will convert into future Human Resource Information Systems (HRIS) which can be used for background verifications, screening profiles, shortlisting candidates and sorting them in folders, contacting candidates in one go by sending messages, creating future candidate pipeline and organising existing candidate pool. Companies invest heavily to grab the right talent. The captcha in future will identify keywords in candidates video resume and enable searches through recruiter interface. AI should also suggest candidates on which keywords should be written according to his skills so that recruiters can easily search them. For example; Right now, candidates are writing micro architecture in so many different ways that recruiter faces difficulty to map all keywords. Some candidates write micro architecture, some may write it as micro-architecture or some may write micro architect. AI should empower candidates with a tool on how recruiters are searching and candidates should be encouraged to use similar keywords for same skills. This would ease the task of a recruiter.

\section{References}

Albert, E. T. (2019). AI in talent acquisition: a review of AI-applications used in recruitment and selection. Strategic HR Review. https://doi.org/10.1108/SHR-04-2019-0024

Geetha, R., \& Bhanu, S. R. D. (2018). Recruitment through artificial intelligence: a conceptual study. International Journal of Mechanical Engineering and Technology, 9(7), 63-70. 
Hmoud, B., \& Laszlo, V. (2019). Will Artificial intelligence Take Over HumanResources Recruitment and Selection. Network Intelligence Studies, 7(13), 21-30.

Keršič, V., Štukelj, P., Kamišalić, A., Karakatić, S., \&Turkanović, M. (2019, June). A blockchain-and AI-based platform for global employability. In International Congress on Blockchain and Applications (pp. 161-168). Springer, Cham. https://doi.org/10.1007/978-3-030-23813-1_20

Krishnakumar, A. (2019). Assessing the Fairness of AI Recruitment systems.

Mujtaba, D. F., \& Mahapatra, N. R. (2019, November). Ethical considerations in ai-based recruitment. In 2019 IEEE International Symposium on Technology and Society (ISTAS) (pp. 1-7). IEEE. https://doi.org/10.1109/ISTAS48451.2019.8937920

Russell-Rose, T., Chamberlain, J., \&Kruschwitz, U. (2019, April). Rethinking 'advanced search': A new approach to complex query formulation. In European Conference on Information Retrieval (pp. 236-240). Springer, Cham. https://doi.org/10.1007/978-3-030-15719-7_31

Russell-Rose, T., \& Chamberlain, J. (2016). Searching for talent: The information retrieval challenges of recruitment professionals. Business Information Review, 33(1), 40-48. https://doi.org/10.1177/0266382116631849

Upadhyay, A. K., \& Khandelwal, K. (2018). Applying artificial intelligence: implications for recruitment. Strategic HR Review. https://doi.org/10.1108/SHR-07-2018-0051

Van Esch, P., Black, J. S., \&Ferolie, J. (2019). Marketing AI recruitment: The next phase in job application and selection. Computers in Human Behavior, 90, 215-222. https://doi.org/10.1016/j.chb.2018.09.009

Wilfred, D. (2018). AI in Recruitment. NHRD Network Journal, 11(2), 15-18. https://doi.org/10.1177/0974173920180204

\section{Copyrights}

Copyright for this article is retained by the author(s), with first publication rights granted to the journal.

This is an open-access article distributed under the terms and conditions of the Creative Commons Attribution license (http://creativecommons.org/licenses/by/4.0/) 